\title{
Gauge Model With Massive Gravitons
}

\author{
Ning $\mathrm{Wu}^{*}$ \\ Institute of High Energy Physics, P.O.Box 918-1, Beijing 100039, P.R.China ${ }^{\dagger}$
}

October 31, 2018

PACS Numbers: 11.15.-q, 04.60.-m, 95.35.+d

Keywords: gauge field, quantum gravity, dark matter and dark energy.

\begin{abstract}
Gauge theory of gravity is formulated based on principle of local gauge invariance. Because the model has strict local gravitational gauge symmetry, gauge theory of gravity is a perturbatively renormalizable quantum model. However, in the original model, all gauge gravitons are massless. We want to ask that whether there exists massive gravitons in Nature? In this paper, we will propose a gauge model with massive gravitons. The mass term of gravitational gauge field is introduced into the theory without violating the strict local gravitational gauge symmetry. Massive gravitons can be considered to be possible origin of dark energy and dark matter in the Universe.
\end{abstract}

*email address: wuning@mail.ihep.ac.cn

${ }^{\dagger}$ mailing address 
It is generally believed that four kinds of fundamental interactions in Nature are gauge interactions and can be described by gauge field theory. From theoretically point of view, gauge principle plays a fundamental role in particle's interactions theory. We know that in Yang-Mills theory, if the system has strict local gauge symmetry, all gauge field must be massless[1]. But, according to experimental results, some gauge bosons are massive[2]. Up to now, there are mainly two mechanisms to introduce the mass term of gauge field. One is spontaneously symmetry breaking and Higgs mechanism [3, 4, 5, 6, 7, 8, 9, 10], which is well-known in constructing the standard model $[11,12,13]$. So, in this mechanism, the introduction of the mass of gauge field violate the local gauge symmetry of the system. Another mechanism is proposed by $\mathrm{N} . \mathrm{Wu}$ in literature[14]. In this mechanism, two sets of gauge field are introduced into the model simultaneously. The biggest advantage of this mass generation mechanism is that the mass term of gauge fields does not violate the local gauge symmetry of the Lagrangian. This model can also be applied to unified electroweak interactions and the unified electroweak theory based on this mechanism has the similar dynamics for low energy electroweak interactions, but contains no Higgs particle[15], which will become a prospective theory for unified electroweak interactions if Higgs particles do not exist in Nature.

Gauge theory of gravity is proposed based on gauge principle[16, 17, 18, 19]. It is known that gauge treatment of gravity was suggested immediately after the gauge theory birth itself $[20,21,22,23,24,25]$. In the traditional gauge treatment of gravity, Lorentz group is localized, and the gravitational field is not represented by gauge potential, but represented by metric field. The theory is non-renormalizable. Gauge theory of gravity is proposed in physics picture of gravity. The model has strict gravitational gauge symmetry. After localization of gravitational gauge group, the gravitational field appears as the corresponding gauge potential. One advantage of gauge theory of gravity is that, in this new theory, four different kinds of fundamental interactions in Nature can be formulated in the same manner, so they can be unified together[26, 27, 28]. In this model, we will apply the mass generation mechanism of gauge field which is preposed in literature [14] to gravitational gauge theory and to introduce the mass term of gravitational gauge field withoug violating the local gauge symmetry of the lagrangian.

In gauge theory of gravity, the most fundamental quantity is gravitational gauge field. In order to introduce mass term of gravitational gauge fields, we need two sets of gravitational gauge fields simultaneously. Suppose that the first set of gauge fields is denoted as $C_{\mu}^{\alpha}$, and the second set of gauge fields is denoted as $C_{2 \mu}^{\alpha}$. For gauge theory of gravity, the symmetry of gravitational interactions is selected to be gravitational gauge symmetry and the corresponding gauge group is selected to be gravitational gauge group. Details on gravitational gauge symmetry and gravita- 
tional gauge group can be found in leterature[18, 19]. Under gravitational gauge transformation, two gravitational gauge fields transform as

$$
\begin{gathered}
C_{\mu}(x) \rightarrow C_{\mu}^{\prime}(x)=\hat{U}_{\epsilon}(x) C_{\mu}(x) \hat{U}_{\epsilon}^{-1}(x)+\frac{i}{g} \hat{U}_{\epsilon}(x)\left(\partial_{\mu} \hat{U}_{\epsilon}^{-1}(x)\right), \\
C_{2 \mu}(x) \rightarrow C_{2 \mu}^{\prime}(x)=\hat{U}_{\epsilon}(x) C_{2 \mu}(x) \hat{U}_{\epsilon}^{-1}(x)-\frac{i}{\alpha g} \hat{U}_{\epsilon}(x)\left(\partial_{\mu} \hat{U}_{\epsilon}^{-1}(x)\right),
\end{gathered}
$$

where $\alpha$ is a constant parameter and $\hat{U}_{\epsilon}(x)$ is the gravitational gauge transformation operator $[16,17,18,19]$

$$
\hat{U}_{\epsilon}(x)=E^{-i \epsilon^{\beta} \cdot \hat{P}_{\beta}} .
$$

Gravitational gauge fields $C_{\mu}(x)$ and $C_{2 \mu}(x)$ are vectors in gravitational Lie algebra[18, 19], so they can be expanded as

$$
\begin{gathered}
C_{\mu}(x)=C_{\mu}^{\alpha}(x) \hat{P}_{\alpha}, \\
C_{2 \mu}(x)=C_{2 \mu}^{\alpha}(x) \hat{P}_{\alpha} .
\end{gathered}
$$

Correspondingly, there are two gauge covariant derivatives,

$$
\begin{gathered}
D_{\mu}=\partial_{\mu}-i g C_{\mu}(x), \\
D_{2 \mu}=\partial_{\mu}+i \alpha g C_{2 \mu}(x),
\end{gathered}
$$

and two different strengths of gauge fields,

$$
\begin{gathered}
F_{\mu \nu}=\frac{1}{-i g}\left[\begin{array}{lll}
D_{\mu} & , & D_{\nu}
\end{array}\right], \\
F_{2 \mu \nu}=\frac{1}{i \alpha g}\left[D_{2 \mu}, \quad D_{2 \nu}\right] .
\end{gathered}
$$

The explicit forms of field strengths are

$$
\begin{gathered}
F_{\mu \nu}=\partial_{\mu} C_{\nu}(x)-\partial_{\nu} C_{\mu}(x)-i g C_{\mu}(x) C_{\nu}(x)+i g C_{\nu}(x) C_{\mu}(x), \\
F_{2 \mu \nu}=\partial_{\mu} C_{2 \nu}(x)-\partial_{\nu} C_{2 \mu}(x)+i \alpha g C_{2 \mu}(x) C_{2 \nu}(x)-i \alpha g C_{2 \nu}(x) C_{2 \mu}(x) .
\end{gathered}
$$

Field strengths $F_{\mu \nu}$ and $F_{2 \mu \nu}$ are also vectors in gravitational Lie algrbra, so they can be expanded in terms of generators of gravitational gauge group

$$
\begin{gathered}
F_{\mu \nu}=F_{\mu \nu}^{\gamma} \hat{P}_{\gamma}, \\
F_{2 \mu \nu}=F_{2 \mu \nu}^{\gamma} \hat{P}_{\gamma},
\end{gathered}
$$


where $F_{\mu \nu}^{\gamma}$ and $F_{2 \mu \nu}^{\gamma}$ are component field strengths. The explicit forms of component strengths are

$$
\begin{gathered}
F_{\mu \nu}^{\gamma}=\partial_{\mu} C_{\nu}^{\gamma}-\partial_{\nu} C_{\mu}^{\gamma}-g C_{\mu}^{\beta} \partial_{\beta} C_{\nu}^{\gamma}+g C_{\nu}^{\beta} \partial_{\beta} C_{\mu}^{\gamma}, \\
F_{2 \mu \nu}^{\gamma}=\partial_{\mu} C_{2 \nu}^{\gamma}-\partial_{\nu} C_{2 \mu}^{\gamma}+\alpha g C_{2 \mu}^{\beta} \partial_{\beta} C_{2 \nu}^{\gamma}-\alpha g C_{2 \nu}^{\beta} \partial_{\beta} C_{2 \mu}^{\gamma} .
\end{gathered}
$$

Using eq.(1-2), we can obtain the following transformation properties,

$$
\begin{aligned}
& D_{\mu}(x) \rightarrow D_{\mu}^{\prime}(x)=\hat{U}_{\epsilon} D_{\mu}(x) \hat{U}_{\epsilon}^{-1}, \\
& D_{2 \mu}(x) \rightarrow D_{2 \mu}^{\prime}(x)=\hat{U}_{\epsilon} D_{2 \mu}(x) \hat{U}_{\epsilon}^{-1}, \\
& F_{\mu \nu} \rightarrow F_{\mu \nu}^{\prime}=\hat{U}_{\epsilon} F_{\mu \nu} \hat{U}_{\epsilon}^{-1} \\
& F_{2 \mu \nu} \rightarrow F_{2 \mu \nu}^{\prime}=\hat{U}_{\epsilon} F_{2 \mu \nu} \hat{U}_{\epsilon}^{-1} \\
&\left(C_{\mu}+\alpha C_{2 \mu}\right) \rightarrow\left(C_{\mu}^{\prime}+\alpha C_{2 \mu}^{\prime}\right)=\hat{U}_{\epsilon}\left(C_{\mu}+\alpha C_{2 \mu}\right) \hat{U}_{\epsilon}^{-1} .
\end{aligned}
$$

Matrix $G$ is an important quantity in gauge theory of gravity, whose definition is $[16,17,18,19]$

$$
G=\left(G_{\mu}^{\alpha}\right)=\left(\delta_{\mu}^{\alpha}-g C_{\mu}^{\alpha}\right) .
$$

Its inverse matrix is denoted as $G^{-1}$,

$$
G^{-1}=\frac{1}{I-g C}=\left(G_{\alpha}^{-1 \mu}\right)
$$

They satisfy the following relations,

$$
\begin{aligned}
G_{\mu}^{\alpha} G_{\alpha}^{-1 \nu} & =\delta_{\mu}^{\nu}, \\
G_{\beta}^{-1 \mu} G_{\mu}^{\alpha} & =\delta_{\beta}^{\alpha} .
\end{aligned}
$$

It can be proved that

$$
D_{\mu}=G_{\mu}^{\alpha} \partial_{\alpha}
$$

In order to construct a gravitational gauge invariant lagrangian, $J(C)$ is an important factor. In this paper, it will select to be[16, 17, 18, 19]

$$
J(C)=\sqrt{-\operatorname{det}\left(g_{\alpha \beta}\right)},
$$

where

$$
g_{\alpha \beta} \triangleq \eta_{\mu \nu}\left(G^{-1}\right)_{\alpha}^{\mu}\left(G^{-1}\right)_{\beta}^{\nu}
$$


The Lagrangian of the system is

$$
\begin{aligned}
\mathcal{L}_{0}= & -\frac{1}{4} \eta^{\mu \rho} \eta^{\nu \sigma} g_{\beta \gamma} F_{\mu \nu}^{\beta} F_{\rho \sigma}^{\gamma}-\frac{1}{4} \eta^{\mu \rho} \eta^{\nu \sigma} g_{\beta \gamma} F_{2 \mu \nu}^{\beta} F_{2 \rho \sigma}^{\gamma} \\
& -\frac{m^{2}}{2\left(1+\alpha^{2}\right)} \eta^{\mu \nu} g_{\beta \gamma}\left(C_{\mu}^{\beta}+\alpha C_{2 \mu}^{\beta}\right)\left(C_{\nu}^{\gamma}+\alpha C_{2 \nu}^{\gamma}\right),
\end{aligned}
$$

where $m$ is a constant mass parameter. The action is defined by

$$
S=\int \mathrm{d}^{4} x J(C) \mathcal{L}_{0}
$$

Using relations $(16-20)$ and the following relation

$$
\int \mathrm{d}^{4} x J \cdot\left(\hat{U}_{\epsilon} f(x)\right)=\int \mathrm{d}^{4} x f(x)
$$

where $J=\operatorname{det}\left(\frac{\partial(x-\epsilon(x))^{\beta}}{\partial x^{\alpha}}\right)$ is the Jacobian of the corresponding transformations and $f(x)$ is an arbitrary function[16, 17, 18, 19], it is easy to prove that the action $S$ has local gravitational gauge symmetry.

From eq.(28), we can see that there is mass term of gravitational gauge fields. In order to obtain the eigenstates of mass matrix, a rotation is needed

$$
\begin{gathered}
C_{3 \mu}=\cos \theta C_{\mu}+\sin \theta C_{2 \mu}, \\
C_{4 \mu}=-\sin \theta C_{\mu}+\cos \theta C_{2 \mu},
\end{gathered}
$$

where $\theta$ is given by

$$
\cos \theta=1 / \sqrt{1+\alpha^{2}}, \quad \sin \theta=\alpha / \sqrt{1+\alpha^{2}} .
$$

After these transformations, the lagrangian $\mathcal{L}_{0}$ will becomes

$$
\mathcal{L}_{0}=-\frac{1}{4} \eta^{\mu \rho} \eta^{\nu \sigma} g_{\beta \gamma} F_{30 \mu \nu}^{\beta} F_{30 \rho \sigma}^{\gamma}-\frac{1}{4} \eta^{\mu \rho} \eta^{\nu \sigma} g_{\beta \gamma} F_{40 \mu \nu}^{\beta} F_{40 \rho \sigma}^{\gamma}-\frac{m^{2}}{2} \eta^{\mu \nu} g_{\beta \gamma} C_{3 \mu}^{\beta} C_{3 \nu}^{\gamma}+\mathcal{L}_{I},
$$

where $\mathcal{L}_{I}$ contains all interaction terms of gravitational gauge fields and

$$
\begin{aligned}
& F_{30 \mu \nu}^{\alpha}=\partial_{\mu} C_{3 \nu}^{\alpha}-\partial_{\nu} C_{3 \mu}^{\alpha}, \\
& F_{40 \mu \nu}^{\alpha}=\partial_{\mu} C_{4 \nu}^{\alpha}-\partial_{\nu} C_{4 \mu}^{\alpha} .
\end{aligned}
$$

So, the gravitational gauge field $C_{3 \mu}$ is massive whose mass is $m$ while gravitational gauge field $C_{4 \mu}$ is kept massless. 
Gravitational gauge field $C_{3 \mu}$ is massive. If its mass is very large, it will have no contribution to the long range gravitational force. Long range gravitational force completely comes from the contribution of gravitational gauge field $C_{4 \mu}$ and obeys inverse square law. But, if the mass term of gravitational gauge field $C_{3 \mu}$ is extremely small, gravitational gauge field $C_{3 \mu}$ will also contribute some to the middle range gravitational force. Its force range $l$ is about

$$
l \sim \frac{h c}{m}
$$

where $h$ is the plank constant and $c$ is the speed of light. So, if $m$ is about $2 \times 10^{-7}$ $\mathrm{eV}$, its force range $l$ will be about one meter. The gravitational force carried by gravitatioanl gauge field $C_{3 \mu}$ decreases exponentially. In this case, the inverse square law of gravitational force in middle range will be violated. But long range gravitational force still obey inverse square law.

The existence of massive gravitons in Nature is very important for cosmology. Because the coupling constant for gravitational interactions is weak and the massive gravitons only take part in gravitational interactions, the massive gravitons must be a relative stable particle in Nature and they have very weak coupling with ordinary matter. They have no direct electromagnetic interactions with photons, so they radiate no photons. Because they take part in gravitational interactions, they will contribute some to the average energy density of the Universe, and affect the global structure and evolution of the Universe. In a ward, they can not be seen, but they are heavy and affect motions of ordianry celestial objects and global structure and evolution of our Universe. So, if they indeed exist, it must be dark matter or dark energy in the Universe. According to recent results of cosmological observations, especially from Cosmic Microwave Bakground (CMB) temperature anisotropies, our Universe is spacially flat, consists of mainly dark matter and dark energy[29]. A natural origin for dark energy and dark energy is to regard them as gravitons.

If massive gravitons exist in Nature, it will be extremely hard for us to directly detect them in experiments, for they can not be seen in any experiments and they have very weak coupling with ordianry matter. For a single massive graviton, because its mass is very small, its gravitational interactions with any experimental instruments is almost zero, it will leave no signal to any experimental instruments. So, the only possibility to prove the existence of mass graviton is to find evidence in astrophysical and cosmological phenomenon.

Because Higgs mechanism violates local gauge symmetry of the system and we know that gravitational gauge symmetry is a strict symmetry, Higgs mechanism can not be used to introduce the mass term of gravitational gauge fields. 


\section{References}

[1] C.N.Yang, R.L.Mills, Phys Rev 96 (1954) 191 .

[2] Particle Data Group, Euro. Phys. J. C3 (1998) 1.

[3] J. Goldstone, Nov. Cim. 19 (1961) 154.

[4] Y Nambu, G.Jona-Lasinio, Phys.Rev. 122 (1961)34.

[5] J.Goldstone, A.Salam, S.Weinberg. Phys.Rev.127. (1962) 965.

[6] P.W.Higgs, Phys.Lett.12(1964) 132.

[7] F.Englert, R.Brout Phys.Rev.Lett. 13 (1964) 321.

[8] G.S.Guralnik, C.R.Hagen, T.W.B.Kibble Phys.Rev.Lett 13 (1964) 585.

[9] P.W.Higgs Phys.Rev. 145 (1966) 1156.

[10] S.Weinberg, Phys.Rev. D7 (1973) 1068.

[11] S.Glashow, Nucl Phys 22(1961) 579.

[12] S.Weinberg, Phys Rev Lett 19 (1967) 1264.

[13] A.Salam, in Elementary Particle Theory, eds.N.Svartho Forlag, Stockholm,1968) .

[14] Ning Wu, Commun.Theor.Phys., 36(2001) 169.

[15] Ning Wu, Commun.Theor.Phys., 38 (2002): 577-582.

[16] Ning Wu, Commun.Theor.Phys., 38 (2002): 151-156.

[17] Ning Wu, "Quantum Gauge Theory of Gravity", talk given at Meeting of the Devision of Particles and Fields of American Physical Society at the College of William \& Mery(DPF2002), May 24-28, 2002, Williamsburg, Virgia, USA.

[18] Ning Wu, "Gauge Theory of Gravity", hep-th/0109145.

[19] Ning Wu, "Renormalizable Quantum Gauge Theory of Gravity", hepth/0207254.

[20] R.Utiyama, Phys.Rev.101 (1956) 1597.

[21] A.Brodsky, D.Ivanenko and G. Sokolik, JETPH 41 (1961)1307; Acta Phys.Hung. 14 (1962) 21. 
[22] T.W.Kibble, J.Math.Phys. 2 (1961) 212.

[23] D.Ivanenko and G.Sardanashvily, Phys.Rep. 94 (1983) 1.

[24] F.W.Hehl, J.D.McCrea, E.W.Mielke and Y.Ne'eman Phys.Rep. 258 (1995) 1171.

[25] F.W.Hehl, P. Von Der Heyde, G.D.Kerlick, J.M.Nester Rev.Mod.Phys. 48 (1976) 393-416.

[26] Ning Wu, Commun.Theor.Phys., 38 (2002): 322-326.

[27] Ning Wu, Commun.Theor.Phys., 38 (2002): 455-460.

[28] Ning Wu, Commun.Theor.Phys., 39 (2003): 561-568.

[29] Wayne Hu and Scott Dodelson, Annu.Rev.Astron.and Astrophys., 40 (2002) $1-51$. 